\title{
More than just a urinary catheter: achieving hemostasis using Foley catheter in deep cerebral cavities-technical nuances
}

\author{
Venkatesan Sanjeevi ${ }^{*}$ and V. R. Roopesh Kumar
}

\begin{abstract}
Background: Achieving hemostasis during neurosurgical procedures within deep seated tumors is of paramount importance. Chemical methods like using ORC and gel foam are preferred to bipolar cautery as bipolar cautery causes thermal injury to the normal eloquent surrounding white matter fibers, thereby causing significant morbidities. In addition to the chemical methods, we advocate a new relatively simple mechanical method by using small size Foley catheter inflated with saline can achieve hemostasis in case of deep locating brain tumor surgery with diffuse oozing from the tumor bed and surrounding white matter tissues are of concern. The balloon tamponade effect of the inflated Foley catheter helps in achieving complete hemostasis without damaging the surrounding normal white matter parenchyma.
\end{abstract}

Case presentation: A 52-years-old female admitted with history of progressive drowsiness and altered sensorium. Brain MRI was done showing large right-sided trigonal meningioma. Right parieto-occipital craniotomy was done, through the superior parietal lobe, corticotomy was done, and tumor was reached. Gentle retraction was done using curved blades in between the tumor and normal brain parenchyma. During surgery, following tumor removal, there was a diffuse oozing from tumor bed and the surrounding stretched white matter fibers. Hemostasis was attempted with chemical methods like ORC and gel foam. We avoided bipolar cautery to prevent thermal injury to the normal stretched eloquent white matter, as bleeding was not settled over the period of 45 min using chemical methods. Then, we placed a $10 \mathrm{~F}$ size Foley catheter in the tumor cavity and inflated with $6 \mathrm{ml}$ of saline over the period of 10 min. Prior to Foley placement, we coated ORC over the tumor bed and the surrounding white matter. This achieved hemostasis to a significant extent and the same was repeated for another $10 \mathrm{~min}$, and finally, complete hemostasis was achieved. Postoperative period went uneventful. Patient was discharged with good neurological recovery.

Conclusion: Inflatable Foley catheter balloon is a simple, cost-effective technique for achieving hemostasis in deep white matter tumors in addition to the routinely available hemostatic techniques.

Keywords: Foley catheter, Thermal injury, Oxidized regenerated cellulose (ORC)

\section{Background}

Achieving hemostasis during neurosurgical procedures within deep seated tumors is of paramount importance. There are various options available to achieve hemostasis in deep seated brain tumor surgery like electrical, chemical, and mechanical methods $[1,2]$. Chemical methods like using oxidized regenerated cellulose

*Correspondence: sanjeevivenkatesan@gmail.com

Department of Neurosurgery, Apollo Proton Cancer Centre (APCC), Chennai 600041 , India

Springer Open
(ORC) and gel foam are preferred to bipolar cautery as bipolar cautery causes thermal injury to the normal eloquent surrounding white matter fibers, thereby causing significant morbidities [3-5]. In addition to the chemical methods, we advocate a new relatively simple mechanical method by using small size Foley catheter inflated with saline which can achieve hemostasis in case of deep locating brain tumor surgery with diffuse oozing from the tumor bed and surrounding white matter tissues are of concern. The balloon tamponade effect of the inflated 
Foley catheter helps in achieving complete hemostasis without damaging the surrounding normal white matter parenchyma.

\section{Case presentation}

A 52-year-old female admitted with history of progressive drowsiness and altered sensorium. Brain magnetic resonance imaging (MRI) was done showing large rightsided trigonal meningioma (Fig. 1). Right parietooccipital craniotomy was done, through the superior parietal lobe, corticotomy was done, and tumor was reached. Using judicious microsurgical technique, tumor debulking was done using ultrasonic aspirator. Gentle retraction was done using curved blades in between the tumor and normal brain parenchyma. During surgery, following tumor removal, there was a diffuse oozing from tumor bed and the surrounding stretched white matter fibers. Hemostasis was attempted with chemical methods like ORC and gel foam. We avoided bipolar cautery to prevent thermal injury to the normal stretched eloquent white matter, as bleeding was not settled over the period of 45 min using chemical methods. Then, we placed a $10 \mathrm{~F}$ size Foley catheter in the tumor cavity and inflated with $6 \mathrm{ml}$ of saline over the period of $10 \mathrm{~min}$. Prior to Foley placement, we coated ORC over the tumor bed and the surrounding white matter (Fig. 2). This achieved hemostasis to a significant extent and the same was repeated for another 10 min and finally complete hemostasis was achieved. Postoperative period went uneventful. Patient was discharged with good neurological recovery.

\section{Discussion}

Tumors in a deep seated location pose significant surgical challenges. For excising these difficult tumors meticulous microsurgical debulking of the tumors, piece meal removal of tumors using ultrasonic aspirator, gentle retraction with curved blades, vascular pedicle control, and newer hemostatic agents. Usually, bleeding in deep cavity causes challenges in various ways like brain bulge, difficult visualization, and acute hydrocephalus (bleeding into ventricle) all of which can lead to post op significant morbidity; the technical nuances in achieving hemostasis in deeper location include simple techniques like warm saline irrigation, gel foam in case of venous bleeding, and ORC in case of capillary and small arteriolar bleeding. Obvious pulsatile bleeding from larger vessels requires bipolar coagulation. The newer technique include flowable gelatin which is of help in difficult to reach location [6, 7]. Fibrin glue which is primarily used as dural sealant is also of help in difficult bleeding scenario especially in cavernous sinus bleeding $[2,8-10]$. Nevertheless, most of these methods require good coagulation profile of the patient. If deranged coagulation, the appropriate replacement of blood component is mandatory to achieve hemostasis. We describe a relatively simple, cost-effective technique which is described in other location but not in the brain. Small size Foley catheter after inflating the balloon with saline can cause circumferential, uniform tamponade which does not cause any permanent damage to neurons or white matter and is reversible. They can be utilized especially in deep location where surrounding white matter can be used as the gradient. Diffuse oozing along the tumor bed
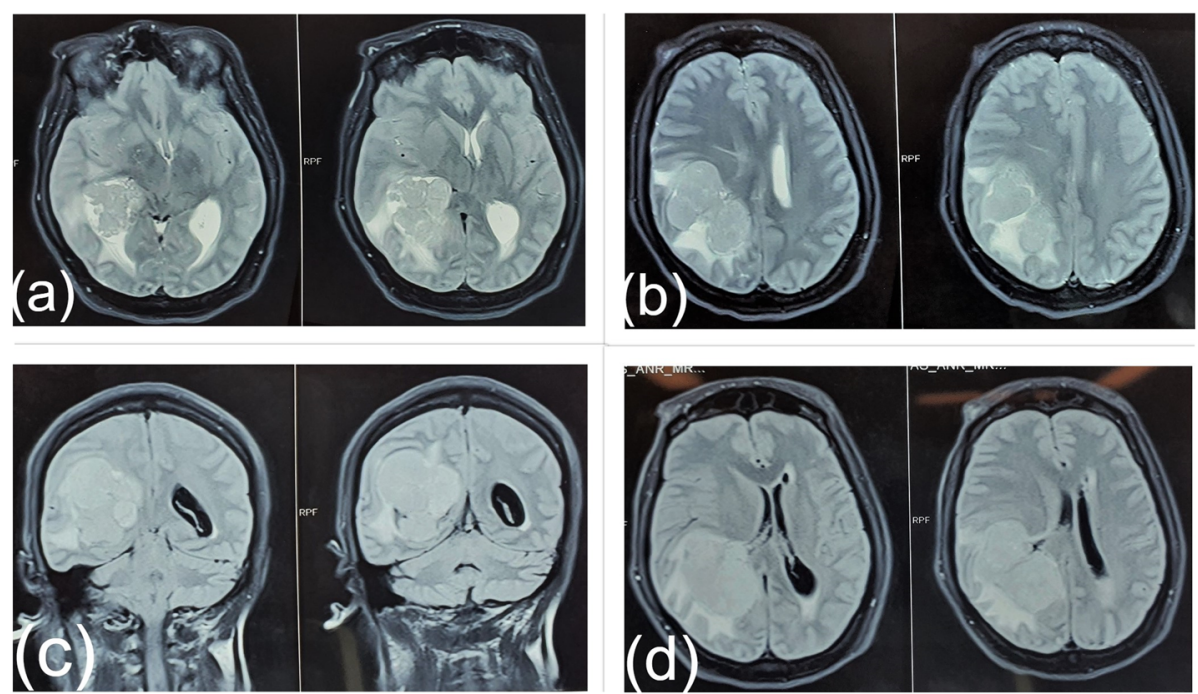

Fig. 1 a, b MRI scan brain T2WI axial image shows large trigonal meningioma. c, d Brain MRI FLAIR image shows trigonal meningioma with mass effect 

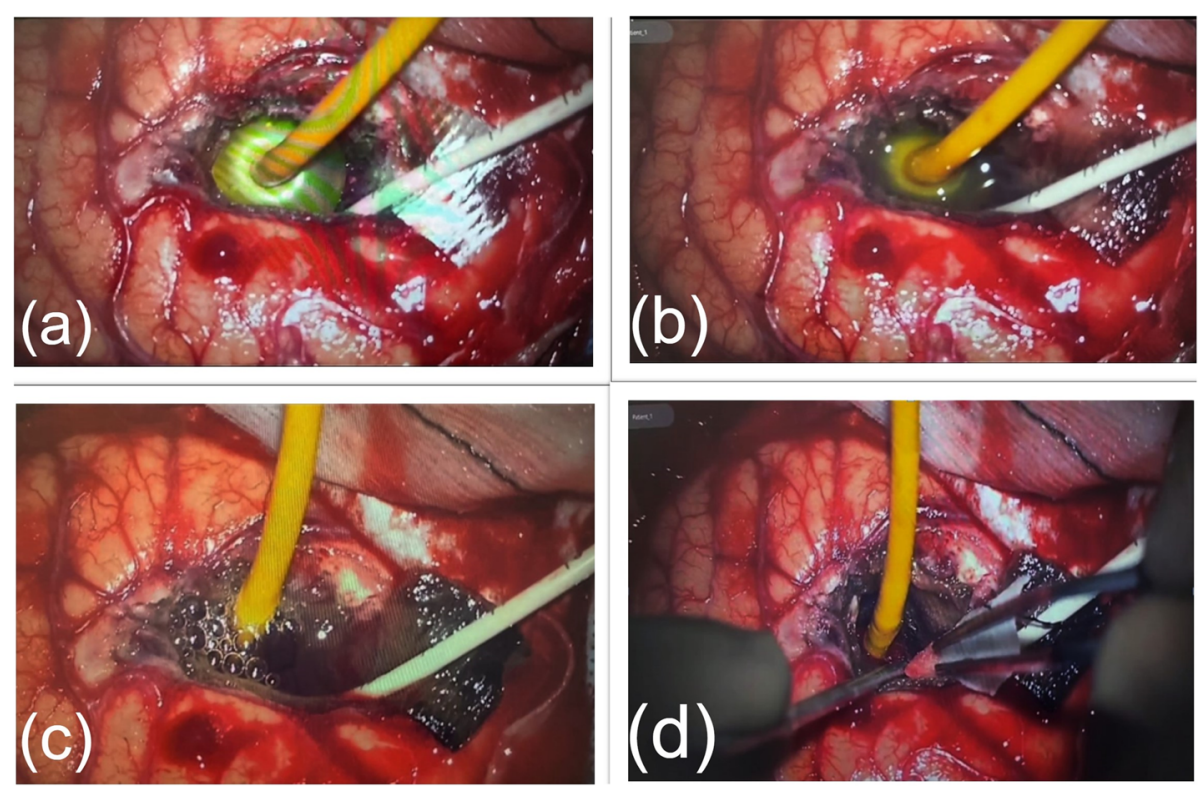

Fig. 2 a, b Placement of Foley catheter into the tumor cavity after tumor resection and inflation of bulb. c, $\mathbf{d}$ Deflation of Foley bulb and complete hemostasis achieved

especially near the eloquent cortex would be the ideal scenario.

\section{Conclusion}

Inflatable Foley catheter balloon is a simple, costeffective technique for achieving hemostasis in deep white matter tumors in addition to the routinely available hemostatic techniques.

\section{Abbreviations}

ORC: Oxidized regenerated cellulose; MRI: Magnetic resonance imaging

\section{Acknowledgements}

The authors thank the patient of this study

\section{Authors' contributions}

VS and RK performed the clinical part of this study, and all authors have read and approved the final manuscript.

\section{Funding}

This study had no funding from any resource.

\section{Availability of data and materials}

The data supporting our findings can be found with the corresponding author and can be contacted through the following e-mails: sanjeevivenkatesan@gmail.com, roops1975@gmail.com

\section{Declarations}

Ethics approval and consent to participate Not applicable

\section{Consent for publication}

Available, written informed consent was obtained from patient for publication.

\section{Competing interests}

The authors declare that they have no conflict of interest.
Received: 10 August 2020 Accepted: 10 June 2021

Published online: 25 October 2021

\section{References}

1. Spotnitz WD. Hemostats, sealants, and adhesives: a practical guide for the surgeon. Am Surg. 2012;78:1305-21 Hemostats, sealants, and adhesives are necessary elements in the modern surgeon's toolbox. The salient elements of safety, efficacy, usability, and relative costs of these agents are described as well as when and how to best use these materials.

2. Minato N, Katayama Y, Yunoki J, Kawasaki H, Satou H. Hemostatic effectiveness of a new application method for fibrin glue, the "rub-andspray method", in emergency aortic surgery for acute aortic dissection. Ann Thorac Cardiovasc Surg. 2009;15(4):265-71.

3. Speyer M, Joe J, Davidson JM, Ossoff RH, Reinisch L. Thermal injury patterns and tensile strength of canine oral mucosa after carbon dioxide laser incisions. Laryngoscope. 1996;106(7):845-50. https://doi.org/10.1097/ 00005537-199607000-00012.

4. Liboon J, Funkhouser W, Terris DJ. A comparison of mucosal incisions made by scalpel, CO2 laser, electrocautery, and constantvoltage electrocautery. Otolanyngol Head Neck Surg. 1997;116(3):379-85. https://doi.org/10.1016/S0194-5998(97)70277-8.

5. Carew JF, Ward RF, LaBruna A, Torzilli PA, Schley WS. Effects of scalpel, electrocautery, and $\mathrm{CO} 2$ and KTP lasers on wound healing in rat tongues. Lanyngoscope. 1998; 108(3):373-80. https//doi.org/10.1097/00005537-199803000-00012.

6. Galanakis I, Vasdev N, Soomro N. A review of current hemostatic agents and tissue sealants used in laparoscopic partial nephrectomy. Rev Urol. 2011;13:131-8.

7. Reuthebuch $\mathrm{O}$, Lachat ML, Vogt P, Schurr U, Turina M. FloSeal: a new hemostyptic agent in peripheral vascular surgery. Vasa. 2000;29(3):204-6. https://doi.org/10.1024/0301-1526.29.3.204.

8. Achneck HE, Sileshi B, Jamiolkowski RM, Albala DM, Shapiro ML, Lawson JH. A comprehensive review of topical hemostatic agents. Ann Surg. 2010; 251(2):217-28. https://doi.org/10.1097/SLA.0b013e3181c3bcca.

9. Jackson MR. Fibrin sealants in surgical practice: an overview. Am J Surg. 2001;182(2):1S-7S. https://doi.org/10.1016/S0002-9610(01)00770-X.

10. Spotnitz WD, Prabhu R. Fibrin sealant: tissue adhesive - review and update. J Long Term Med Implants. 2005;15(3):245-70. https://doi.org/10.1615/ JLongTermEffMedlmplants.v15.i3.20.

\section{Publisher's Note}

Springer Nature remains neutral with regard to jurisdictional claims in published maps and institutional affiliations. 